\title{
A superposition principle for Siegert resonant states*
}

\author{
David L. Huestis ${ }^{\dagger}$ \\ Arthus Amos Noyes Laboratory of Chemical Physics, ${ }^{\star}$ California Institute of Technology, Pasadena, \\ California 91125 \\ (Received 5 May 1975)
}

The utilization of the Siegert resonant states as a natural basis for the scattering process has been constrained by the lack of a convergent superposition formula. This paper presents such an expansion of the exact scattering wavefunction over the $s$-wave Siegert resonant states for finite range potentials. Any integral property of the scattering wavefunction can then be calculated from the resonances.

\section{INTRODUCTION}

Since the early days of the quantum theory of scattering, metastable states have served as an appealing explanation for the structure observed in cross sections. In the discussion of some specific physical interaction, say $\pi+p$ or $e^{-}+H_{2}$, these compound states of the target and projectile are associated with a pole of the $S$ matrix. Breit and Wigner ${ }^{1}$ gave a simple model formula that describes the cross section near such a resonance:

$$
\sigma \simeq \frac{\pi}{k^{2}} \frac{\Gamma^{2}}{\left(E-E_{\mathrm{res}}\right)^{2}+\frac{1}{4} \Gamma^{2}}
$$

where $\Gamma$ is the width of the resonance and $2 / \Gamma$ is the lifetime of the resonant state. This important one-level formula is a satisfactory description when the widths are narrow compared with the energy separation between the resonances. In the more general case one expects to use a superposition of Breit-Wigner terms with relative phases chosen in some yet unspecified manner.

Regge ${ }^{2}$ provided a remarkably simple albeit slowly convergent ${ }^{3}$ expression for the $S$ matrix by a Weierstrass-Hadamard infinite product over all the resonances

$$
S(k)=\exp \left(-2 i k r_{0}\right) \prod_{n} \frac{\left(k_{n}+k\right)}{\left(k_{n}-k\right)}
$$

where $r_{0}$ is the actual radius of the potential. Regge's formula remains correct regardless of where the resonances are located, provided the potential is of finite range. This formula gives an expansion over the resonances from the purely mathematical properties of the $S$ matrix, but it camouflages the physically appealing role of the resonances as transient intermediates for the scattering process.

The objective here is more than just to describe the shape of cross section or $S$ matrix in terms of dispersion formulae such as (1) and (2). Rather, the basic ideas are: (i) the resonances are compound states, (ii) the incoming particle has some amplitude to populate each of these states, and (iii) it is the subsequent decay of the res onant states that is observed

$$
\psi_{\text {in }} \rightarrow \quad \sum_{n} \alpha_{n} \varphi_{n}+\psi_{\text {out }}
$$

incoming $\rightarrow$ resonances + unscattered.

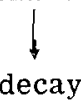

The problem at hand is to characterize the resonance energies and resonant states as manifestations of the potential, then to describe the scattering process as mediated by the resonances. ${ }^{4}$

In particular, we need to develop some kind of superposition principle for resonant states:

$$
\psi_{\text {given }}=\sum_{n} \alpha_{n} \varphi_{n} \text {. }
$$

This would presumably illustrate that once a given wavefunction is expanded, any property could be written in terms of the simpler and decoupled properties of the resonant states themselves:

$$
\operatorname{property}\left[\psi_{\text {given }}\right]-\operatorname{properties}\left[\varphi_{n}\right] \text {. }
$$

Such a superposition principle we now set out to find.

\section{THE RESONANT STATES}

Kapur and Peierls ${ }^{5}$ suggested that the eigenvalue problem

$$
\begin{aligned}
& -\frac{\hbar^{2}}{2 m} \theta_{n}^{\prime \prime}(r)+V(r) \theta_{n}(r)=E_{n} \theta_{n}(r), \quad 0 \leqslant r \leqslant r_{0}, \\
& \theta_{n}(0)=0, \quad \theta_{n}^{\prime}\left(r_{0}\right)=i k \theta_{n}\left(r_{0}\right)
\end{aligned}
$$

for a potential of finite range $r_{0}$ would provide the $s$ wave resonance energies as eigenvalues $E_{n}=E_{\text {res }}+(i)$ 2) $\Gamma$. It is crucial to note that each solution behaves as an outgoing plane wave at the right-hand boundary, but that $k$ is chosen to match the momentum of the incoming particle we wish to describe. The resulting eigenfunctions $\left[\theta_{n}\right]$ are called the Kapur-Peierls states. A superposition formula was derived from these states for the exact scattering wavefunction

$$
\psi_{k} \rightarrow \sum_{n} \beta_{n}(k) \theta_{n} \text {. }
$$

Whence a dispersion formula for the transition matrix was available:

$$
\begin{aligned}
T= & \frac{\hbar^{2}}{2 m} \exp \left(-2 i k r_{0}\right) \sum_{n} \frac{\theta_{n}^{2}\left(r_{0}\right)}{\left(E_{n}-E\right) N_{n}}-\frac{1}{k} \sin k r_{0} \\
& \times \exp \left(-i k r_{0}\right),
\end{aligned}
$$

where $N_{n}$ is the normalization integral $N_{n}=\int_{0}^{r_{0}} \theta_{n}^{2}(r) d r$.

This model has attracted attention for two reasons. First, it appears to offer a technique for finding the resonances for a variety of potentials, ${ }^{6}$ even for problems with many particles. ${ }^{7}$ Second, the expansions (7a) and (7b) over a complete ${ }^{8}$ set of resonant states appears to provide a description of all of the properties and structures of the scattering process in terms of the

Copyright (c) 1975 American Institute of Physics 
resonances. Unfortunately there is one principal difficulty. The Kapur-Peierls formula (7b) suggests that the $S$ matrix should have poles at the Kapur-Peierls energies. Although these energies may in some cases be close to the actual poles, they are not coincident. Indeed the poles of the $S$ matrix are characteristic of the potential itself, whereas the Kapur-Peierls energies depend on the incoming momentum $k$, and in addition upon the choice of $r_{0}$. This is true even when $r_{0}$ is chosen outside the actual range of the potential!

Siegert ${ }^{9}$ noticed this difficulty and suggested an alternative eigenvalue problem:

$$
\begin{aligned}
& -\frac{\hbar^{2}}{2 m} \varphi_{n}^{\prime \prime}+V \varphi_{n}=E_{n} \varphi_{n}, \quad \frac{\hbar^{2}}{2 m} k_{n}^{2}=E_{n}, \\
& \varphi_{n}(0)=0, \quad \varphi_{n}^{\prime}\left(r_{0}\right)=i k_{n} \varphi_{n}\left(r_{0}\right) .
\end{aligned}
$$

We call this the Siegert eigenvalue problem; the eigenvalues are called the Siegert energies or Siegert poles; and the eigenfunctions are called the Siegert states. Use of the resonance momentum in the plane-wave boundary condition alleviates some of the unsatisfactory features of the Kapur-Peierls description. In particular one can easily show ${ }^{9}(i)$ that the Siegert energies are at the poles of the $S$ matrix, (ii) that they are independent of both the actual scattering energy and the joining radius (as long as it is chosen outside the actual potential), and finally (iii) that the bound states are just special solutions of the Siegert equations (8).

Siegert also found a one-level formula that gives the correct residue of the $S$ matrix at the resonance pole and reduces to the Breit-Wigner formula when the width $\Gamma$ is small. However, he was unable to provide a complete description of the scattering process due to the lack of an expansion over the resonant states. Any attempt to remedy this situation is complicated by the nonorthogonality of the Siegert states (which arises from having the boundary condition depend on the eigenvalue), and by the rather slow convergence of the residues. These are the difficulties to be overcome below.

\section{THE TWO-COMPONENT WAVEFUNCTION}

We first rewrite the Siegert $s$-wave eigenvalue problem in a more dimensionless form:

$$
\begin{aligned}
& -\varphi_{n}^{\prime \prime}+U \varphi_{n}=k_{n}^{2} \varphi_{n}, \quad \varphi_{n}(0)=0, \quad \varphi_{n}^{\prime}\left(r_{0}\right)=i k_{n} \varphi_{n}\left(r_{0}\right), \\
& k_{n}^{2}=\left(2 m / \hbar^{2}\right) E_{n}, \quad U=\left(2 m / \hbar^{2}\right) V .
\end{aligned}
$$

Unfortunately, the literature of such problems with the eigenvalue in the boundary conditions is meager. However, Friedman ${ }^{10}$ discusses two similar problems:

$$
-\varphi^{\prime \prime}=k^{2} \varphi, \quad \varphi(0)=0, \quad \varphi^{\prime}(1)=k^{2} \varphi(1),
$$

and

$$
-\varphi^{\prime \prime}=k^{2} \varphi, \quad \varphi(0)=0, \quad \varphi^{\prime}(1)=k \varphi(1) .
$$

A further description of these two and other related systems can be found in Ref. 3 .

An important suggestion of the work in Refs. 3 and 10 is that we consider writing the solutions to Eq. (9) as two-component wavefunctions

$$
\Phi_{n}=\left[\begin{array}{l}
\varphi_{n}^{(1)}(r) \\
\varphi_{n}^{(2)}(r)
\end{array}\right]
$$

Then the eigenvalue problem is rewritten as

$$
\mathbf{L} \Phi_{n}=\left[\begin{array}{l}
\varphi_{n}^{(2)}(r) \\
\varphi_{n}^{(1)^{\prime}}(r)+\int_{r}^{r_{0}} U(x) \varphi_{n}^{(1)}(x) d x
\end{array}\right]=i k_{n} \Phi_{n},
$$

with the boundary conditions

$$
\varphi_{n}^{(n)}(0)=0, \quad \varphi_{n}^{(1)}\left(r_{0}\right)=\varphi_{n}^{(2)}\left(r_{0}\right)
$$

The upper component $\varphi_{n}^{(1)}(r)$ from a solution $\Phi_{n}$ of $\mathrm{Eq}$. (12a) can be seen to be a solution to (9) by differentiation of the lower components of (12a)

$$
\varphi_{n}^{(1)^{\prime \prime}}-U \varphi_{n}^{(1)}=i k_{n} \varphi_{n}^{(2)^{\prime}}=-k_{n}^{2} \varphi_{n}^{(1)} \text {. }
$$

In this two-component space we define the inner product by

$$
\langle\boldsymbol{\Psi}, \boldsymbol{\Phi}\rangle=\int_{0}^{r_{0}}\left(\psi^{(1)} \varphi^{(1)}-\psi^{(2)} \varphi^{(2)}\right)
$$

where

$$
\boldsymbol{\Psi}=\left[\begin{array}{l}
\psi^{(1)} \\
\psi^{(2)}
\end{array}\right], \quad \boldsymbol{\Phi}=\left[\begin{array}{l}
\varphi^{(1)} \\
\varphi^{(2)}
\end{array}\right] .
$$

With the boundary conditions (12b) and the inner product (14) we can find the adjoint operator $\mathrm{L}^{+}$:

$$
\mathbf{L}^{\dagger} \boldsymbol{\Phi}=\left[\begin{array}{l}
\varphi^{(2)^{\prime}}(\gamma)-U(r) \int_{0}^{r} \varphi^{(2)}(x) d x \\
\varphi^{(1)^{\prime}}
\end{array}\right] .
$$

This defines a new set of adjoint eigenvectors (with the old eigenvalues)

$$
\mathbf{L}^{\dagger} \Phi_{n}^{\dagger}=i k_{n} \Phi_{n}^{\dagger} \text {. }
$$

We notice further that if the boundary conditions (12b) are satisfied, then

$$
\begin{aligned}
& \mathbf{L}^{2} \Phi_{n}=\left[\begin{array}{l}
\varphi_{n}^{(1)^{\prime \prime}}-U \varphi_{n}^{(1)} \\
\varphi_{n}^{(2)^{\prime \prime}}+\int_{r}^{r 0} U(x) \varphi_{n}^{(2)^{\prime}}(x) d x
\end{array}\right]=-k_{n}^{2} \Phi_{n}, \\
& \left(\mathbf{L}^{+}\right)^{2} \Phi_{n}^{\dagger}=\left[\begin{array}{l}
\varphi_{n}^{\dagger(1)^{\prime \prime}}-U \varphi_{n}^{+(1)} \\
\varphi_{n}^{\dagger(2)^{\prime \prime}}-\left\{U(r) \int_{0}^{r} \varphi_{n}^{\dagger(2)}\right\}
\end{array}\right]=-k_{n}^{2} \Phi_{n}^{+} .
\end{aligned}
$$

Equations (12), (15), and (17) show that the upper components of $\left\{\Phi_{n}\right\}$ and $\left\{\boldsymbol{\Phi}_{n}^{+}\right\}$can be chosen to be the same and that this function, $\varphi_{n}=\varphi_{n}^{(1)}=\varphi_{n}^{\dagger(1)}$, is the physical Siegert resonant wavefunction. The lower components are yet without physical interpretation.

Toward the goal of a superposition principle, it will be necessary to evaluate the inner product of $\Phi_{n}^{+}$with $\Phi_{l}$. As might be expected the choice of the form of the inner product and the relation of $\mathrm{L}^{+}$to $\mathrm{L}$ make this especially simple:

$$
i\left(k_{n}-k_{l}\right)\left\langle\Phi_{n}^{\dagger}, \Phi_{l}\right\rangle=\left\langle\mathbf{L}^{\dagger} \Phi_{n}^{\dagger}, \Phi_{l}\right\rangle-\left\langle\Phi_{n}^{\dagger}, \mathbf{L} \Phi_{l}\right\rangle=0,
$$

so that either $\left\langle\Phi_{n}^{+}, \Phi_{l}\right\rangle=0$, or $k_{l}=k_{n}$. The solutions are in general nondegenerate, ${ }^{4 a}$ so we have

$$
\left\langle\Phi_{n}^{\dagger}, \Phi_{l}\right\rangle=N_{l} \delta_{n l},
$$


where $\delta_{n l}$ is the Kronecker delta and $N_{l}$ may be considered the (not necessarily real) norm of the state. As is customary the normalization constant could be absorbed into the wavefunction, yielding $N_{l}=1$, except that a Siegert state could possibly have a norm of zero. Indeed we anticipate null vectors and degenerate roots to appear simultaneously ${ }^{11}$ but only for special values of the potential strength.

Now we have our superposition principle. To expand a given two-component vector

$$
\mathbf{F}=\left[\begin{array}{l}
f^{(1)} \\
f^{(2)}
\end{array}\right] \rightarrow \sum_{n} \alpha_{n} \Phi_{n},
$$

we only need to calculate

$$
\alpha_{l}=\frac{1}{N_{l}}\left\langle\Phi_{l}^{\dagger}, \mathbf{F}\right\rangle=\frac{1}{N_{l}} \int_{0}^{r_{0}}\left(\varphi_{l}^{\dagger(1)} f^{(1)}-\varphi_{l}^{\dagger(2)} f^{(2)}\right) .
$$

\section{EXPANSION OF THE PHYSICAL SCATTERING STATE}

For any energy there exists a function $\psi_{k}$ satisfying

$$
-\psi_{k}^{\prime \prime}+U \psi_{k}=k^{2} \psi_{k}, \quad \psi_{k}(0)=0 .
$$

This $\left(\psi_{k}\right)$ is the exact scattering wavefunction. The principal goal of this work is to find a set of coefficients $\alpha_{l}(k)$ such that

$$
\psi_{k}(r)=\sum_{n} \alpha_{n}(k) \varphi_{n}(r)
$$

that is, to express $\psi_{k}$ as a superposition of Siegert resonances.

The function $\psi_{k}$ specifies only the upper component of the vector to be expanded:

$$
\Psi_{k}=\left[\begin{array}{l}
\psi_{k} \\
?
\end{array}\right]=\sum_{n} \alpha_{n}(k) \Phi_{n} .
$$

At this point it does not seem too important what lower component is used. Physically, we are only interested in the upper component, at least for now. There are two difficulties. First, even though the upper component of $\Psi_{k}$ seems independent of the lower one, the expansion coefficients will depend on both components through Eq. (21). This is the same as saying that there are many sequences of coefficients $\left\{\alpha_{n}\right\}$ that give the same expanded upper component, or as noted by More and Gerjuoy, ${ }^{13}$ the Siegert basis is overcomplete. The series (23) will converge very slowly or not at all if the lower component is not chosen with care. Second, the expansion (23) will be essentially useless if we actually have to evaluate integrals involving the yet unknown $\psi_{k}$. If the Siegert states are in fact a natural basis for the scattering problem, the stationary wavefunctions, $\psi_{k}$ would be expressable directly. That is what Kapur and Peierls tried to do.

A little experimentation with Fourier series convinces one that the attempt to expand a function that does not satisfy the same boundary conditions as the basis set produces slow and nonuniform convergence (see also Ref. 3). The problem is at its worst when expanding in Siegert states. The Siegert states, even when normalized to unity are not bounded with respect to $k_{n}$. The point in the interval at which convergence will be the most difficult to obtain will be $r=r_{0}$, since there the
Siegert states will in general by the largest $\left(\left|\varphi_{n}\left(r_{0}\right)\right| \sim \exp \left[\left|\operatorname{Im}\left(k_{n}\right)\right| r_{0}\right]\right)$.

It was found ${ }^{3}$ that an expansion over Siegert states [Eq. (20)] will not converge at all unless at least three conditions are met:

$$
\begin{aligned}
& \text { (1) } f^{(1)}(0)=0, \\
& \text { (2) } f^{(1)}\left(r_{0}\right)=f^{(2)}\left(r_{0}\right), \\
& \text { (3) } f^{(1)^{\prime}}\left(r_{0}\right)=f^{(2)}\left(r_{0}\right) .
\end{aligned}
$$

These conditions amount to making the vector to be expanded as continuous as possible at the right-hand boundary. For the problem at hand, the lower component of $\Psi_{k}$ is uniquely defined if $\Psi_{k}$ is required to satisfy both (25) and the very useful relation

$$
\mathbf{L}^{2} \Psi_{k}=-k^{2} \Psi_{k} \text {. }
$$

This equation is a relatively natural choice since its upper component is identical to Eq. (22).

All of this gives

$$
\boldsymbol{\Psi}_{k}=\left[\begin{array}{l}
\psi_{k} \\
a \int^{r} \psi_{k}+b \int^{r} \bar{\psi}_{k}+c
\end{array}\right]
$$

where $\bar{\psi}_{k}$ satisfies the conditions

$$
-\bar{\psi}_{k}^{\prime \prime}+U \bar{\psi}_{k}=k^{2} \bar{\psi}_{k}, \quad \bar{\psi}^{\prime}(0)=0,
$$

and $a, b$, and $c$ are constants to be determined from (25) and (26). Having chosen $\Psi_{k}$ we need only evaluate

$$
\alpha_{n}(k)=\left\langle\boldsymbol{\Phi}_{n}^{\dagger}, \boldsymbol{\Psi}_{k}\right\rangle / N_{n} \text {. }
$$

To evaluate $\alpha_{n}$ we use a trick much like that used by Kapur and Peierls: From (16) we have

$$
\Phi_{n}^{\dagger}=\frac{\mathbf{L}^{\dagger 2}+k^{2}}{\left(i k_{n}\right)^{2}+k^{2}} \Phi_{n}^{\dagger},
$$

so that

$$
\alpha_{n}=\frac{1}{\left(k^{2}-k_{n}^{2}\right) N_{n}}\left\{k^{2}\left\langle\Phi_{n}^{\dagger}, \Psi_{k}\right\rangle+\left\langle\mathbf{L}^{\dagger 2} \Phi_{n}^{\dagger}, \Psi_{k}\right\rangle\right\}
$$

But now

$$
\begin{aligned}
\left\langle\mathbf{L}^{\dagger} \boldsymbol{\Phi}_{n}^{\dagger}, \boldsymbol{\Psi}_{k}\right\rangle & =\left\langle\mathbf{L}^{\dagger} \boldsymbol{\Phi}_{n}^{\dagger}, \mathbf{L} \boldsymbol{\Psi}_{k}\right\rangle-\left[\left[\mathbf{L}^{\dagger} \boldsymbol{\Phi}_{n}^{\dagger}\right]^{(1)} \psi_{k}^{(2)}-\left[\mathbf{L}^{\dagger} \boldsymbol{\Phi}_{n}^{\dagger}\right]^{(2)} \psi_{k}^{(1)}\right]_{0}^{\dagger c} \\
& =\left\langle\mathbf{L}^{\dagger} \boldsymbol{\Phi}_{n}^{\dagger}, \mathbf{L} \boldsymbol{\Psi}_{k}\right\rangle+0 .
\end{aligned}
$$

Next

$$
\begin{aligned}
\left\langle\mathbf{L}^{\dagger} \boldsymbol{\Phi}_{n}^{\dagger}, \mathbf{L} \boldsymbol{\Psi}_{k}\right\rangle & =\left\langle\boldsymbol{\Phi}_{n}^{\dagger}, \mathbf{L}^{2} \boldsymbol{\Psi}_{k}\right\rangle-\left[\varphi_{n}^{\dagger(1)}\left[\mathbf{L} \boldsymbol{\Psi}_{k}\right]^{(2)}-\varphi_{n}^{\dagger(2)}\left[\mathbf{L} \boldsymbol{\Psi}_{k}\right]^{(1)}\right]_{0}^{r_{0}} \\
& =\left\langle\boldsymbol{\Phi}_{n}^{\dagger}, \mathbf{L}^{2} \boldsymbol{\Psi}_{k}\right\rangle-b \bar{\psi}_{k}(0) \varphi_{n}^{\dagger(2)}(0)
\end{aligned}
$$

Finally, using (31) and (26)

$$
\alpha_{n}(k)=\frac{-b \bar{\psi}_{k}(0) \varphi_{n}^{\dagger(2)}(0)}{\left(k^{2}-k_{n}^{2}\right) N_{n}}=\frac{-b \bar{\psi}_{k}(0) \varphi_{n}^{\prime}(0)}{\left(k^{2}-k_{n}^{2}\right) N_{n}\left(-i k_{n}\right)} .
$$

The term $-b \bar{\psi}_{k}(0)$ is unknown until we have solved for the exact scattering wavefunction. But it is independent of $n$, so it will be replaced by $A(k)$ and treated as a normalization constant to be determined later or ignored altogether. Thus the expansion formula becomes

$$
\psi_{k}(r)=A(k) \sum_{n} \frac{1}{\left(k^{2}-k_{n}^{2}\right)} \frac{\varphi_{n}^{\prime}(0)}{\left(-i k_{n}\right) N_{n}} \varphi_{n}(r) .
$$

This is our superposition formula. It specifies how 
TABLE I. Some Siegert $s-$ wave eigenvalues for the finite square well, depth $U=-\frac{1}{2}$, radius $r_{0}=1$.

\begin{tabular}{lclcl}
\hline \hline$n^{(a)}$ & $\operatorname{Re} k_{n}$ & $\operatorname{Im} k_{n}$ & $\operatorname{Re} k_{n}^{\prime}$ & $\operatorname{Im} k_{n}^{\prime}$ \\
\hline 0 & 0.0 & -1.6506 & 0.0 & -1.4914 \\
1 & 4.0993 & -2.6555 & 4.1423 & -2.6279 \\
2 & 7.4268 & -3.1404 & 7.4553 & -3.1284 \\
3 & 10.661 & -3.4642 & 10.682 & -3.4573 \\
4 & 13.859 & -3.7083 & 13.876 & -3.7038 \\
5 & 17.040 & -3.9044 & 17.054 & -3.9012 \\
6 & 20.210 & -4.0684 & 20.222 & -4.0660 \\
7 & 23.373 & -4.2094 & 23.384 & -4.2075 \\
\hline \hline
\end{tabular}

(a) The corresponding resonances with $n<0$ satisfy $k_{n}=-k_{-n}^{*}$.

the exact stationary state $\psi_{k}$ is to be expanded over the Siegert resonant states $\varphi_{n}$. Aside from the trivial normalization factor $A(k)$, the coefficients have a simple dependence on $k$,

$$
\alpha_{n}(k) \sim \frac{1}{k^{2}-k_{n}^{2}} .
$$

The only significant property of $A(k)$ is that $A(k)$ $\sim\left(k^{2}-k_{l}^{2}\right)$ as $k \rightarrow k_{l}$, so that $\psi_{k} \rightarrow \varphi_{l}$ as $k \rightarrow k_{l}$ just as we would want.

We note as well that the nonphysical lower components of both $\boldsymbol{\Psi}_{k}$ and $\Phi_{n}$ have disappeared from the formula. Having this principle of superposition, we can now proceed to calculate the exact wavefunction at any energy from Siegert states. The various properties of the wavefunction can also be calculated, such as the phase shift for which we would use the LippmannSchwinger equation to avoid convergence difficulties in taking derivatives.

\section{THE SQUARE WELL}

The above description of the scattering process is not an attempt to give yet another dispersion formula. It

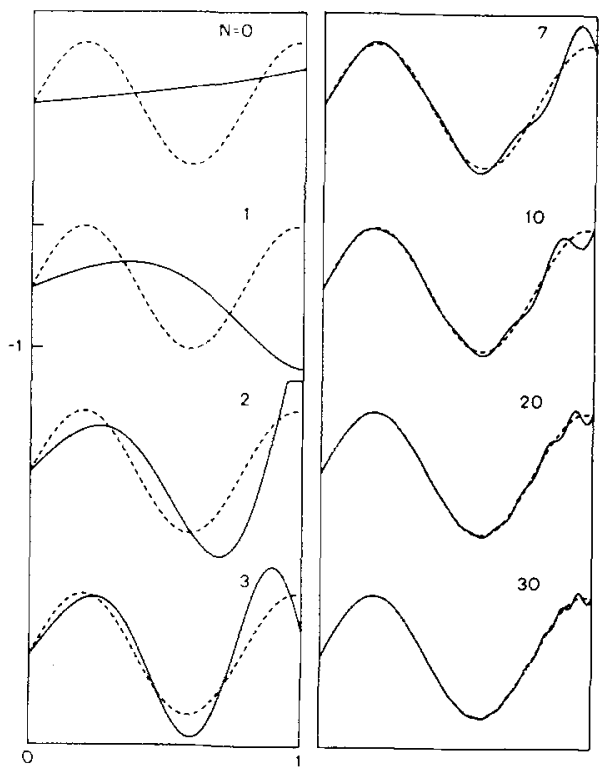

FIG. 1. Expansion $\psi_{k}(\gamma)=\Sigma N_{-N} \alpha_{n} \varphi_{n}(r)$ over square well Siegert states for $k=8$. Dashed line indicates the exact solution; the solid lines represent the partial expansion to $\pm N$. The imaginary parts of the partial expansions cancel when the terms $\alpha_{n} \varphi_{n}$ and $\alpha_{-n} \varphi_{-n}$ are included together. was hoped that the Siegert states would provide a practi cal, discrete (to a good approximation finite), and calculable basis with which to describe scattering events. Along this line it is necessary to consider an example. In fact, the example has been the fulcrum of the theory.

Specifically we consider

$$
U(r)=\left\{\begin{array}{cc}
-\frac{1}{2}, & 0 \leqslant r \leqslant r_{0}=1 \\
0, & r>r_{0}
\end{array} .\right.
$$

This is a rather weak potential; the resonances are far from the real axis; and therefore the theory should be hard pressed. The Siegert states are

$$
\begin{aligned}
& \Phi_{n}=\left[\begin{array}{l}
\sin k_{n}^{\prime} r \\
-i \frac{k_{n}}{k_{n}^{\prime}} \cos k_{n}^{\prime} r+i\left(\frac{k_{n}}{k_{n}^{\prime}}-\frac{k_{n}^{\prime}}{k_{n}}\right) \cos k_{n}^{\prime}
\end{array}\right], \\
& \boldsymbol{\Phi}_{n}^{+}=\left[\begin{array}{l}
\sin k_{n}^{\prime} r \\
-i \frac{k_{n}^{\prime}}{k_{n}} \cos k_{n}^{\prime} r
\end{array}\right]
\end{aligned}
$$

where $k_{n}^{\prime 2}=k_{n}^{2}-U=k_{n}^{2}+\frac{1}{2}$. The resonance eigenvalues satisfy [from Eq. (12b)]

$$
k_{n}^{\prime} \cos k_{n}^{\prime}=i k_{n} \sin k_{n}^{\prime},
$$

which can be solved by Newton's method or as in Ref. 4a. A few of the calculated Siegert resonance eigenvalues are given in Table $I$.

To accomplish the expansion as in Eq. (34), we will need to know the norm, $N_{n}=\left\langle\Phi_{n}^{\dagger}, \Phi_{n}\right\rangle=1+i / k_{n}$, and $\varphi_{n}^{\prime}(0)=k_{n}^{\prime}$. So then

$$
\psi_{k}(r)=A(k) \sum_{n} \frac{1}{k^{2}-k_{n}^{2}} \frac{k_{n}^{\prime}}{\left(-i k_{n}\right)\left(1+i / k_{n}\right)} \sin k_{n}^{\prime} r .
$$

Comparisons of partial expansions of this uniformly convergent series ${ }^{14}$ with the known wavefunctions are shown in Figs. 1 and 2. The appropriate value of $A(k)$ was separately calculated in each case.

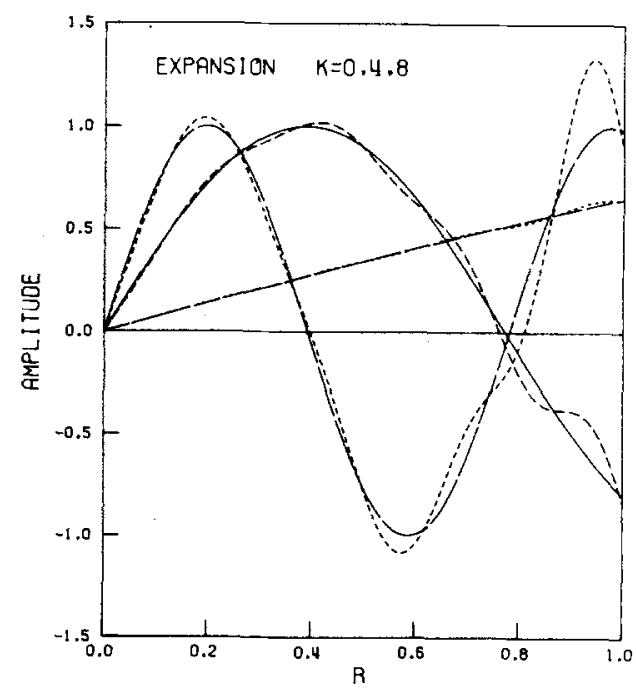

FIG. 2. Square well Siegert expansion $\psi_{k}(r)=\sum_{-6}^{6} \alpha_{n} \varphi_{n}(r)$ for $k$ $=0$ (medium dashes), $k=4$ (long dashes), and $k=8$ (solid). Shorter dashed lines represent the partial expansions. 


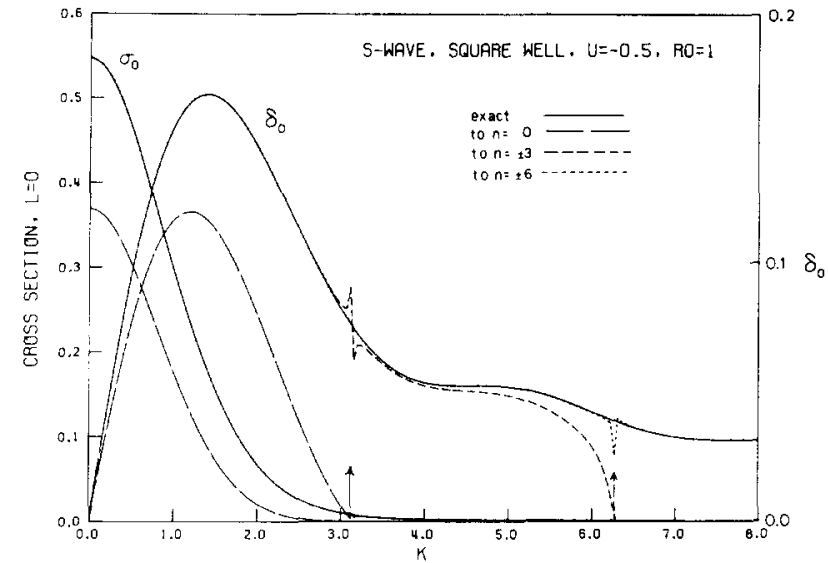

FIG. 3. Siegert expansion of $\delta_{0}$ and $\sigma_{0}$. The singular behavior near $k=\pi, 2 \pi$ is due to the division by $\sin \left(k r_{0}\right)$ in the Lippmann-Schwinger evaluation of the phase shift. As indicated, the solid lines show the exact values, the dashed lines show partial expansions.

As a final illustration, we have in Fig. 3 plots of the phase shift $\delta_{0}$, calculated from the Lippmann-Schwinger equation ${ }^{14}$ along with the cross section $\sigma_{0}=\left(\pi / k^{2}\right)$ $\times \sin ^{2} \delta_{0}$, for various numbers of exapnsion terms. The exact result is shown for comparison. We note the excellent agreement even with only a few terms in the expansion.

\section{CONCLUSION}

This paper has discussed an expansion of the $s$-wave scattering wavefunction over the Siegert resonant states, which is uniformly convergent at least for the finite square well. This is not to be viewed as the derivation of a dispersion formula but rather as the beginning of a calculational device. If the Siegert states turn out to resemble the bound states as much as it now appears that they do, then all of the powerful and successful approximation techniques that have been used in bound state quantum mechanics may then be used for scattering states. Specifically in view is the calculation of the Siegert states for a molecular system, such as $N_{2}+e$, by some kind of self-consistent field procedure. The scattering problem is then just a rigorous expansion over the boundlike Siegert states. No fictitious "potential scattering" term or matching at the boundary would be required.

The sense of accomplishment that might be expected must be deflated by one important observation. All of the results presented apply, as yet, only to potentials of finite range (or at best dying faster than any exponential). Few physical problems have potentials of such short range. But resonances are observed nevertheless. The experience gained in this research indicates (to the author at least) that the work now in progress on Siegert states for long-range potentials will bring similar results. At that point the theory will have come to the motivation: electron-molecule scattering.

\section{ACKNOWLEDGMENTS}

It is my pleasure to acknowledge the helpful suggestions of Professor J.K. Knowles and Professor T.C. McGill. In particular I would like to thank Professor W. A. Goddard III for his unfailing interest, encouragement, enthusiasm, and support, not to mention many discussions and suggestions.

* Partially supported by National Science Foundation (Grant GP-1 5423) and by the President's Fund Grant (PF -013) of the California Institute of Technology. Taken in part from a thesis submitted 19 September 1972 to the Division of Chemistry and Chemical Engineering in partial fulfillment of the requirements for the $\mathrm{Ph} . \mathrm{D}$. degree, California Institute of Technology, 1973.

†National Science Foundation Predoctoral Fellow, 1968-72. Present address: Molecular Physics Center, Stanford Research Institute, Menlo Park, California 94025.

†Contribution No. 5085

${ }^{1}$ G. Breit and E. P. Wigner, Phys. Rev. 49, 519 (1936).

${ }^{2} \mathrm{~T}$. Regge, Nuovo Cimento 8, 671 (1958).

${ }^{3} \mathrm{D}$. L. Huestis, Ph. D. thesis, California Institute of Technology (1973). Available from University Microfilms.

${ }^{4} \mathrm{~T}$ he emphasis in this work will be on the wavefunctions for the resonant states. H. M. Nussenzvieg [(a) Nucl. Phys. 11, 499 (1959) and (b) Nuovo Cimento 20, 694 (1961); "Analytic Properties of Nonrelativistic Scattering Amplitudes," Escuela Latino Americana de Fisica, Universidad de México, Mexico, D. F. (1962)] and G. Beck and H. M. Nussenzvieg [(c) Nuovo Cimento 16, 416 (1960)] also capitalized on the resonances as transient intermediates in their development of resonance propagators. See also Ref. 12.

${ }^{5}$ P. L. Kapur and R.E. Peierls, Proc. Roy. Soc. (Lond.) A 166, 277 (1938).

${ }^{6}$ D. T. Birtwistle and A. Herzenberg, J. Phys. B 4, 53 (1971).

${ }^{7}$ A. Herzenberg and F. Mandl, Proc, Roy. Soc. (Lond.) A 274, 253 (1963).

${ }^{8}$ R. E. Peierls, Proc. Camb. Phil. Soc. 44, 242 (1948).

${ }^{9}$ A. J. F. Siegert, Phys. Rev. 56, 750 (1939).

${ }^{10} \mathrm{~B}$. Fiedman, Principles and Techniques of Applied Mathematics (Wiley, New York, 1956), pp. 205-207.

${ }^{11}$ See P.N. Dobson, Jr., J. Math. Phys. 12, 1207 (1971), and Ref. 10 .

${ }^{12}$ R. M. More and E. Gerjuoy, Phys. Rev. A 7, 1288 (1973). ${ }^{13}$ The series is majorized by $n^{-3 / 2}$.

${ }^{14} \mathrm{~T}$. - Y. Wu and T. Ohmura, Quantum Theory of Scattering (Prentice-Hall, Englewood Cliffs, N.J., 1962), p. 64. 